\title{
Comparison of Kato-Katz and Formol-Ether concentration methods for the diagnosis of intestinal helminthic infections among school children of Wonji Shoa town, Eastern Ethiopia: A school based cross-sectional study
}

\author{
Solomon Taye \\ College of Medicine and Health Sciences, Madawalabu University, Bale Goba, Ethiopia
}

\section{Email address:}

salbertnick@yahoo.com

\section{To cite this article:}

Solomon Taye. Comparison of Kato-Katz and Formol-Ether Concentration Methods for the Diagnosis of Intestinal Helminthic Infections among School Children of Wonji Shoa Town, Eastern Ethiopia: A School Based Cross-Sectional Study. American Journal of Health Research. Vol. 2, No. 5, 2014, pp. 271-274. doi: 10.11648/j.ajhr.20140205.18

\begin{abstract}
Background: Intestinal helminthic infections are among the most common infections in the world and are responsible for considerable morbidity and mortality. The diagnosis of these infections commonly relies on the detection of helminth egg or larvae in stool. The objective of this study was to compare the diagnostic performance of kato-katz thick smear and formol-ether concentration methods for the diagnosis of helminthic infections among Wonji Shoa town primary school children. Method: Institution based cross-sectional study design was used. 669 primary school students were selected using simple random sampling technique. Stool samples were examined using formol-ether concentration and kato-katz thick smear techniques. Results were shown in tables, percent and prevalence rates. $\mathrm{P}<0.05$ was considered as statistically significant. Result: The stool concentration method showed that the prevalence of S. mansoni was (28.3\%), A. lumbricoides (15.2\%), Hookworms (12.1\%) and T. trichiura (12.1\%) respectively. Both Taenia species and $H$. nana were equally prevalent (3.0\%). However, Kato-katz method showed a prevalence of $20.2 \%, 8.1 \%$ and $5.8 \%$ for S. mansoni, A. lumbricoides and Hookworms respectively. The sensitivity of the two techniques was statistically significant $(p=0.02)$. Conclusion: Even though the Kato-katz thick smear is the most recommended method for epidemiological study of $S$. mansoni, in this study, it has lower sensitivity than concentration technique in the diagnosis of each type of helminthes. Since human intestinal parasitic infections are high in the study area; mass treatment, snail control and health education were strongly recommended.
\end{abstract}

Keywords: Kato-Katz, Formol-Ether Concentration, Parasitic Infections, Helmithiasis

\section{Introduction}

Intestinal parasites are among the most common human infections which are distributed throughout the world [1]. They cause thousands of avoidable outpatient morbidity and mortality especially; many of school age children suffer from these parasitic infections. They are also the leading cause of gastrointestinal pain, malnutrition, malabsorption, anaemia, mental retardation and other diseases [2]. In Ethiopia, helminthiasis is common and rank first in many parts of the country especially in the rural community [3].

The diagnosis of helminthic infections commonly relies on the detection of helminth eggs or larvae in human stool. It is based on the microscopic observation of parasite eggs in stools, by means of a direct faecal smear, concentration technique, kato-katz thick smear or immunological and molecular approaches [4].

At present, the Kato-Katz parasitologic technique is the most recommended method and widely used copromicroscopic method in epidemiological surveys because of its simplicity and low cost. However, the small amount of stool analyzed (usually $41.7 \mathrm{mg}$ ) decreases the sensitivity Kato-Katz technique to detect eggs whenever they are present at low frequency or appear highly clustered $[5,6]$. The sensitivity of the Kato-Katz method is further compromised by day-to-day and intra specimen variation of helminth egg output, problems related to time delays from fresh fecal sample production, collection in the field and 
processing in the laboratories and rapid over clearing of hookworm eggs [7].

The ether-concentration method is often used for the diagnosis of helminthic infections, particularly in specialized laboratories. Importantly, it allows the concurrent diagnosis of intestinal protozoa and is sometimes used in combination with the Kato-Katz method to enhance diagnostic sensitivity for helminthes. An important feature of the etherconcentration method is that it uses preserved stool samples, fixed in either sodium acetate-acetic acid-formalin (SAF) or diluted formalin, thus allowing sample storage and analysis at later time points [8].

As most African countries, Ethiopia is one of the highly affected country with helminthiasis infection. In Ethiopia, prevalence of helminthiasis varies from place to place and generally it is not well documented. Even though, there is no significant drug resistance problem against helminthiasis infection, accurate diagnosis of helminthiasis is crucial to minimize its prevalence and for effective chemotherapy and control measures.

Wonji Shoa town is found in Oromia regional state, $110 \mathrm{~km}$ away from Addis Ababa, Ethiopia in eastern direction. In this town, previously few studies have done on the prevalence of intestinal parasites and to the best of my knowledge no studies have been done on the comparison of concentration and Kato-katz techniques. Hence, the objective of this study was to compare the diagnostic performance of kato-katz thick smear and formol-ether concentration methods in the diagnosis of helminthic infections.

\section{Methods and Materials}

This cross-sectional study was conducted from AprilMay 2011, in primary school of Wonji Shoa town, east, Ethiopia. Wonji Shoa is a small town located $110 \mathrm{kms}$ away from Addis Ababa in the south east direction.

In Wonji Shoa town primary school, there are a total of 1568 students and 669 of them were selected using simple random sampling (lottery method). The school was stratified to grades and number of study participants were allocated proportionally to each grades based on their number of sections and/or number of students.

The sample size was determined using the single proportion population formula taking the prevalence rate of intestinal helminthic infection at Babile primary school,
Eastern Ethiopia $(\mathrm{P}=27.2 \%)$ [11]. For the calculation a $95 \%$ confidence interval $(\mathrm{Z}), 5 \%$ margin of error $(\mathrm{d})$ and design effect of two (2) was used to allow for multi-stage and stratified sampling. The calculated sample size was 669 including ten percent (10\%) non response rate.

Study participants were instructed how to collect stool samples and provided with labeled clean plastic container, toilet tissue paper and applicator sticks. Each sample was examined using kato-katz thick smear and formol-ether concentration methods. Kato-katz thick smear was prepared immediately after collection and examined within 30 minutes for hookworms' egg at Wonji Shoa town health center laboratory. Enough amounts of stool samples were also preserved in SAF (Sodium acetate-acetic acid-formalin) for concentration technique and examined later on.

Data entry and analysis was done using SPSS version 16 computer software. Data was summarized and presented in a descriptive measure such as table, figures, mean and percent. Comparison analysis was done to rate the diagnostic performances of the two methods. $\mathrm{P}$ value less than 0.05 was considered statistically significant.

The study protocol was approved by Addis Ababa University, Faculty of life sciences, biology department research ethics committee. Before sampling, administrators, students and parents and teachers clearly informed about the objective of the study. All students involved in this study were provided a written informed consent prior to data collection. Furthermore, written informed consent was obtained from each student's family for publication. Infected students were treated appropriately free of charge and health education was given.

\section{Result}

\subsection{Concentration Technique}

Stool examination result showed that, from the total 669 students, $492(73.5 \%)$ of them were infected by at least one parasite. As shown in table 1 , out of the $492 / 669$ positive students, $189 / 669(28.3 \%)$ of them were infected by $S$. mansoni, 101/669(15.2\%) were positive for $A$. lumbricoides. Hookworms and T. trichiura were equally prevalent $(81 / 669(12.1 \%))$. Furthermore, H. nana and Taenia species were equally infected $20 / 669(3 \%)$ students. All the above mentioned parasites were detected singly or by co-infecting with other parasites.

Table 1. Distribution of helminthic parasites among Wonji Shoa town primary school children using Concentration and Kato-katz methods, Eastern Ethiopia, 2011

\begin{tabular}{llll}
\hline Type of Parasite & Formol-ether Concentration Method $(\mathbf{N}=\mathbf{6 6 9})$ & Kato-katz Thick Smear Method $(\mathbf{N}=\mathbf{6 6 9})$ & P-value \\
\hline S. mansoni & $189(28.3)^{*}$ & $135(20.2)$ & \\
A. lumbricoides & $101(15.2)$ & $54(8.1)$ \\
Hookworms & $81(12.1)$ & $39(5.8)$ & \\
T. trichiura & $81(12.1)$ & $34(5.1)$ & \\
H. nana & $20(3.0)$ & $5(0.8)$ & \\
Taenia species & $20(3.0)$ & $5(0.8)$ & 0.02 \\
Total & $492(73.5 \%)$ & $272(40.7 \%)$ & \\
\hline
\end{tabular}

* The numbers in parentheses indicates percent prevalence of each parasite. 
273 Solomon Taye: Comparison of Kato-Katz and Formol-Ether Concentration Methods for the Diagnosis of Intestinal Helminthic Infections among School Children of Wonji Shoa Town, Eastern Ethiopia: A School Based Cross-Sectional Study

\subsection{Kato-katz Thick Smear}

Using Kato-katz thick smear method, from the total 669 , only $272(40.7 \%)$ students were positive for at least one helminthic intestinal infection. Of those infected, $135 / 669(20.2 \%)$ of them infected by $S$. mansoni. Moderately prevalent parasites were A. lumbricoides 54/669(8.1\%), Hookworms 39/669(5.8\%) and T. trichiura $34 / 669(5.1 \%)$. H. nana 5/669(0.8\%) and Taenia species $5 / 669(0.8 \%)$ were the least prevalent parasites using KatoKatz thick smear technique (Table 1).

\subsection{Multiple Parasitic Infections (Poly-Parasitism)}

As shown in table 2, double parasitic infections were detected in $109 / 669(16.3 \%)$ and $49 / 669(7.3 \%)$ of the students using concentration and Kato methods respectively. Using stool concentration method, the most prevalent co- infected parasites were A. lumbricoides and $S$. mansoni $34(5.1 \%)$ followed by $S$. mansoni and Hookworms 27(4.0\%). With Kato method, co-infection of $A$. lumbricoides and S. mansoni 30(4.5\%) were highly prevalent followed by $A$. lumbricoides and $H$. nana $5(0.8 \%)$ and Hookworm and T. trichiura 5(0.8\%).

Table 3 shows the prevalence of triple parasitic infections. The prevalence of triple infection was 42(6.3\%) and $26(3.9 \%)$ using the concentration and Kato methods respectively. T. trichiura, S. mansoni and Hookworms (2\%) was the most frequent triple parasitic infection using concentration method whereas, triple infections with $A$. lumbricoides, T. trichiura and S. mansoni (1.5\%) was the commonest poly-parasitism using Kato thick smear method. Except the triple infections, the diagnostic performances of the two methods were statistically significant at a $\mathrm{p}$ value of $=0.02$ for single and $p=0.003$ for double infections.

Table 2. Distribution of double helminthic parasite infections among Wonji Shoa town primary school children using Concentration and Kato-katz Techniques, Eastern Ethiopia, 2011

\begin{tabular}{lll}
\hline Parasites in Co-infection & Concentration Technique $(\mathbf{N}=669)$ & Kato-katz Thick Smear $(\mathbf{N}=669)$ \\
\hline A. lumbricoides and $S$. mansoni & $34(5.1)$ & $30(4.5)$ \\
A. lumbricoides and Hookworms & $7(1.0)$ & $3(0.5)$ \\
A. lumbricoides and Taenia species & $7(1.0)$ & $3(0.5)$ \\
A. lumbricoides and H. nana & $7(1.0)$ & $5(0.8)$ \\
S. mansoni and Hookworms & $27(4.0)$ & $3(0.5)$ \\
Hookworms and T. trichiura & $20(3.0)$ & $5(0.8)$ \\
T. trichiura and $S$. mansoni & $7(1.0)$ & $49(7.3 \%)$ \\
Total & $109(16.3 \%)$ & 0.003 \\
\hline
\end{tabular}

The numbers in parentheses indicates the percentage of students from the total infected students.

Table 3. Distribution of triple helminthic parasite infections among Wonji Shoa town primary school children using Concentration and Kato-katz Techniques, Eastern Ethiopia, 2011

\begin{tabular}{lll}
\hline Triple Parasitic infections & Concentration Technique $(\mathbf{N}=669)$ & Kato-katz Thick Smear $(\mathbf{N}=\mathbf{6 6 9})$ \\
\hline T. trichiura, S. mansoni and Hookworms & $14(2.0)$ & $8(1.3)$ \\
Hookworms, T. trichiura and Taenia species & $7(1.0)$ & $10(1.5)$ \\
A.lumbricoides, T. trichiura and S. mansoni & $7(1.0)$ & $8(1.3)$ \\
A.lumbricoides, T. trichiura and Hookworms & $7(1.0)$ & \\
S. mansoni, T. trichiura and Taenia species & $7(1.0)$ & $26(3.9 \%)$ \\
Total & $42(6.3 \%)$ & 0.006 \\
\hline
\end{tabular}

-The numbers in parentheses indicates the percentage of students from the total infected students.

\section{Discussion}

Most human intestinal parasites are cosmopolitan in their distribution, but some are endemic in tropical and subtropical regions. Helminthic infection is common mainly in areas where there are low standards of hygiene and sanitation [4, 9, 10, 11]. Various studies have been conducted on the diagnostic performance of kato-katz thick smear and formol-ether concentration methods in different parts of the world. However, no comparison studies were done at the present study area. Thus, this study aimed at determining the diagnostic performance of those two methods in the diagnosis of helminthic infections in Wonji Shoa town.

In the present study, six species of intestinal parasites were found in the faeces of students. Helminthic parasites were found in $492 / 669(73.5 \%)$ of the students, using concentration technique and 272/669(40.7\%) by Kato-katz method. The difference in their diagnostic sensitivity was statistically significant $(p=0.02)$. These differences in diagnostic performance might be due the low sensitivity of the Kato-Katz technique as indicated by other studies or could be due to the technical errors of the two methods.

A similar study done by Kongs et al [5] showed that Kato-katz method has a lower sensitivity than concentration technique. Furthermore, Pontes et al [6] compared the Kato-katz method with polymerase chain reaction (PCR) and showed that Kato-katz has low sensitivity than PCR. However, the result of the present study was in contrary to the study done by Glinz et al [8] who showed that Kato-katz method was more sensitive 
than concentration, Koga agar plate and FLOTAC methods.

In areas where numerous kinds of intestinal parasites found, multiple parasitic infections (poly-parasitism) infections were common. Poly-parasitism were detected in $88(40.7 \%)$ and $45(27.1 \%)$ of the students using concentration and Kato-katz methods respectively, which was a similar result by Feleke et al [9]. Double infections were detected much more than triple infections by both techniques $(16.3 \%$ vs $7.3 \%$ and $6.3 \%$ vs $3.9 \%)$ respectively. The diagnostic performances of the two methods was statistically significant $(p=0.003)$ in detecting double parasitic infections. The prevalence of multiple infections in this study was lower when compared to the previous study by Amare Mengistu [1]. The reason is probably due to environmental conditions and methods of transmission that was favorable for the two parasites to live together or due to a reflection of the local endemicity of the two parasites in the study area.

\section{Conclusion}

In conclusion, comparing the sensitivity of the two methods for the diagnosis of helminthic parasites, usefulness of the Kato-Katz technique appears limited, i.e. its sensitivity was low. Therefore, concentration method can be recommended as a routine technique used in health care centers or in a hospital laboratory. Furthermore, safety and detection of other parasites are better assured by concentration and direct saline methods. Besides, treating infected individuals, continuous cleaning of the canals to eliminate snails, proper use of toilets, personal hygiene and health education are recommended to control schistosomiasis and geohelminthic parasites.

\section{List of Abbreviations}

SAF sodium acetate-acetic acid-formalin

\section{Author's Contribution}

$\mathrm{ST}^{*}$ conceptualized and designed the study and performed the laboratory examinations. ST* also analyzed and interpreted the data, drafted the manuscript and critically reviewed the manuscript.

\section{Acknowledgements}

I would like to thank Professor Berhanu Erko, Addis Ababa University, Aklilu Lemma Institute of Pathobiology for his comment and suggestions. I would also like to thank my colleagues at Madawalabu University for their help and comments during laboratory examinations of the stool samples. Students' parent, teachers and students of Wonji
Shoa town primary school are also fully acknowledged.

\section{References}

[1] Raza, H.H and Sami, R.A. Epidemiolocal Study on Gastrointestinal Parasites. Among Different Sexes, Occupations, and Age Groups in Sulaimani District. J. Duhok Univ. 2009, 12 (1): 317-323.

[2] Garcia, L.S. Diagnostic Medical Parasitology. 5th ed. LSG and associates ASM press, Washington, DC. 2007, pp 266270 .

[3] Getachew H/mariam, Afework Kassu, Gemeda Abebe, Ebba Abate, Demekech Damte, Endris Mekonnen and Fusao Ota. Intestinal parasitic infections in HIV/AIDS and HIV seronegative individuals in a teaching hospital, Ethiopia. Jpn. J. Infect. Dis. 2004, 57: 41-43.

[4] Shimelis Assefa, Berhanu Erko, Girmay Medhin, Zelalem Assefa and Techalew Shimelis Intestinal parasitic infections in relation to HIV/AIDS status, diarrhea and CD4 T-cell count. BMC Infectious Diseases 2009, 9:155doi:10.1186/1471-2334-9-155

[5] Kongs, A., Marks, G., Verle, P. and Stuyft, P.V. The unreliability of the Kato-Katz technique. limits its usefulness for evaluating S. mansoni infections. Trop. Med. Int. Heal. 2001, 6(3):163-169.

[6] Pontes, L.A., Maria C., Katz, O.N., Dias-Neto, E. and Rabello, A. Comparison of a polymerase chain reaction and the kato-katz technique for the diagnosing infection with Schistosoma mansoni. Am. J. Trop. Med. Hyg., 2003, 68(6):652-656.

[7] Tarafder, M.R., Carabin, H., Joseph, L., Balolong J.E., Olveda, R. and McGarvey, S.T. Estimating the sensitivity and specificity of Kato-Katz stool examination technique for detection of hookworms, Ascaris lumbricoides and Trichuris trichiura infections in humans in the absence of a 'gold standard'. Int. J. Parasitol. 2010, 40:399-404.

[8] Glinz, D., Silue', K.D., Knopp, S., Lohourignon, L.K., Yao, K.P., Steinmann, P., Rinaldi, L., Cringoli, G., N'Goran, E.K. and Utzinger, J. Comparing Diagnostic Accuracy of KatoKatz, Koga Agar Plate, Ether-Concentration, and FLOTAC for Schistosoma mansoni and Soil-Transmitted Helminths. 2010, www.plosntds.org

[9] Feleke Moges, Yenew Kebede, Afework Kassu, Getu Degu, Moges Tiruneh and Molla Gedefaw. Infection with HIV and Intestinal Parasites among Sreet Dwellers in Gondar City Northwest Ethiopia. Jpn. J. Infect. Dis. 2006, 59: 400-403.

[10] Amare Mengistu, Solomon Gebre-Selassie and Tesfaye Kassa. Prevalence of intestinal parasitic infections among urban dwellers in southwest Ethiopia. Ethiop. J. Health Dev. 2007, 21(1):12-17.

[11] Girum Tadesse (2005). The prevalence of intestinal helminthic infections and associated risk factors among school children in Babile town, eastern Ethiopia Ethiop. J.Heal. Dev biol. 19(2): 140-147. 